\title{
Field Performance and SSR Analysis of Drought QTL Introgression Lines of Rice
}

\author{
Jung-Pil Suh*, Yong-Jae Won, Eok-Keun Ahn, Jeong-Heui Lee, Woon-Goo Ha, Myeong-Ki Kim, Young-Chan Cho, \\ Eung-Gi Jeong, Bo-Kyeong Kim
}

National Institute of Crop Science, RDA, Suwon 441-857, Republic of Korea

\begin{abstract}
A set of five Korean rice cultivars and seven drought-tolerant indica lines were screened under irrigated non-stress and drought-stressed conditions in the 2011 and 2012 dry seasons at IRRI, Philippines. The drought-stressed experiment received mild to moderate stress. Under drought stress, 'IR86918-B-439-B' had the highest grain yield among all tested lines and 'Hanarembyeo' had the highest grain yield among the five Korean rice cultivars. 'IR86918-B-439-B' also had the highest yield under irrigated non-stress conditions. The grain yield of 'Hanareumbyeo' was similar to 'IR86918-B-439-B' under non-stress conditions. SSR marker analysis was performed using 125 SSR markers for detection of polymorphic markers between the Korean rice cultivars and the drought-tolerant indica lines, and for genetic diversity analysis. Twelve polymorphic markers were identified in the region of three major drought QTLs (DTY1.1, DTY2.2, DTY3.1) in two of the Korean rice cultivars and three of the drought-tolerant lines. These polymorphic markers will be useful as foreground genotyping markers for drought-QTL introgression in Korean rice genetic backgrounds.
\end{abstract}

Keywords Drought tolerance, Genetic diversity, Rice, SSR marker, Yield

\section{INTRODUCTION}

Drought is one of the most severe constraints reducing rice grain yield in rainfed environments. Recent predictions of climate change suggest a further increase in water deficit in the coming years (Wassmann et al. 2009), leading to an increase in the intensity and frequency of drought (Bates et al. 2008). In severe drought years, total rice production losses in drought-prone rice growing countries is very high. In 2004, a severe drought affected more than 2 million ha of total cropped area and over 8 million people in Southeast Asia (Pandey et al. 2007). Drought also affects production on millions of hectares in irrigated areas dependent on surface irrigation, where river flows and water impounded in ponds, tanks, and reservoirs may be insufficient to irrigate the crop in dry years (Maclean et al. 2002). Despite the importance of drought as a major constraint, little success has been achieved in developing drought-tolerant rice cultivars. Most improved cultivars grown in drought- prone areas are the varieties originally bred for irrigated conditions and are highly susceptible to drought (Kumar et al. 2008). Drought stress during the cropping season directly affects grain yield, which is particularly devastating at the reproductive stage (Venuprasad et al. 2009a). As rainfed environments are highly variable, breeding rice for drought-prone rainfed ecosystem requires genotypes that combine high yielding ability with good drought tolerance in addition to tolerance to prevalent biotic stresses. Varieties with high yield potential and improved drought tolerance could reduce risk and help alleviate poverty in drought-prone rice growing areas. Recently drought-tolerant lines such as Apo, Vandana, Way Rarem, N22, and Adaysel have been shown to out-yield susceptible lines such as IR64 and IR72 by several-fold under upland or lowland drought (Venuprasad et al. 2007; Venuprasad et al. 2009b; Bernier et al. 2007; Vikram et al. 2011; Swamy et al. 2011). Crosses between such tolerant and susceptible lines are useful to identify important QTL underlying variation in

Received April 27, 2014; Revised May 10, 2014; Accepted May 27, 2014; Published June 30, 2014

*Corresponding author Jung-Pil Suh, suhjp@korea.kr, Tel: +82-31-290-6710, Fax: +82-31-290-6730 
grain yield under drought stress. QTLs showing large and consistent effects can be utilized through marker-assisted breeding (MAB), a rapid and precise approach to develop high-yielding drought-tolerant rice varieties. To date, several QTLs with large and consistent effects on grain yield under drought stress have been identified at IRRI. Bernier et al. (2007) reported DTY12.1, a QTL on chromosome 12 in the Vandana/Way Rarem population, explaining about $51 \%$ of the genetic variance for yield under severe upland drought stress over two years. A consistent major-effect QTL for grain yield, DTY1.1, was identified to show an effect in several populations. Vikram et al. (2011) detected DTY1.1 consistently over two years in three RIL populations derived from the donor variety N22 crossed to drought-susceptible varieties Swarna, IR64, and MTU1010. Two major-effect QTLs, DTY3.1 and DTY2.1, were identified in an Apo/2*Swarna population explaining $30 \%$ and $15 \%$ of the phenotypic variance, respectively (Venuprasad et al. 2009a). Four major-effect QTLs, DTY2.2, DTY4.1, DTY9.1, and DTY10.1, were identified in Adaysel/IR64-derived populations (Swamy et al. 2011; Swamy et al. 2013). Drought grain yield QTLs DTY2.2, DTY4.1, DTY9.1 and DTY10.1 have been successfully introgressed into an IR64 background and DTY12.1 has been introgressed into a Vandana background.
The drought tolerant IR64-NILs and Vandana-NILs have been successfully evaluated, their yield advantage under drought has been validated, and lines are being processed for release (Kumar et al. 2008; Kumar 2011). The objectives of this study were to evaluate the grain yield of drought tolerant-NILs under irrigated non-stress and drought stress conditions and to detect polymorphic SSR markers between Korean rice cultivars and drought tolerant-NILs to facilitate the introduction of drought tolerance QTLs into Korean rice cultivars.

\section{MATERIALS AND METHODS}

\section{Plant materials}

Five Korean rice cultivars and seven drought tolerant-NILs were used for drought screening along with controls (Table 1 and 2). IR86918-B-223-B, IR86918-B-305-B, IR86918B-382-B and IR86918-B-439-B are nearly isogenic to IR64 and have the drought tolerance QTL, DTY1.1 from N22 (Vikram et al. 2011). IR77298-14-1-2 is also nearly isogenic to IR64 and has drought tolerance QTL, DTY2.2 from Aday Sel (Swamy et al. 2011; Swamy et al. 2013). IR81896-B-B-431 is nearly isogenic to Swarna and has the drought tolerance QTL, DTY3.1 from Apo (Venuprasad et

Table 1. List of Korean rice varieties for QTL introgression.

\begin{tabular}{lccc}
\hline \hline \multicolumn{1}{c}{ Variety } & Ecotype & Remarks & Grain characteristics \\
\hline Jinmibyeo & Japonica & Termperate and tropical adaptable & Non-glutinous \\
Unkwangbyeo & Japonica & High yielding in Korea & Non-glutinous \\
Hanareumbyeo & Tong-il & High yielding in Korea & Non-glutinous \\
Gayabyeo & Tong-il & High compatibility & Non-glutinous \\
Sangnambatbyeo & Japonica & upland rice in Korea & Glutinous, long-grain \\
\hline
\end{tabular}

Table 2. List of drought tolerant QTL-NILs developed from International Rice Research Institute.

\begin{tabular}{lcccc}
\hline \hline \multicolumn{1}{c}{ Variety (GID\#) } & Genetic background & Ecotype & QTLs & QTL source \\
\hline IR 86918-B-223-B (\#2963514) & IR64 & Indica & DTY & N22 \\
IR 86918-B-305-B (\#2963523) & IR64 & Indica & DTY & N22 \\
IR 86918-B-382-B (\#2963599) & IR64 & Indica & DTY & N22 \\
IR 86918-B-439-B (\#2963506) & IR64 & Indica & DTY & N22 \\
IR 77298-14-1-2 (\#909971) & IR64 & Indica & DTY & Aday sel \\
IR 81896-B-B-431 (\#2155763) & Swarna & Indica & DTY & Apo \\
IR 84984-83-15-18-B-B (\#2719923) & Vandana & Indica & DTY 12.1 & Way Rarem \\
\hline
\end{tabular}


al. 2009a). IR84984-83-15-18-B-B is nearly isogenic to Vandana with the drought tolerance QTL, DTY12.1 from Way Rarem (Bernier et al. 2007). Seeds of these drought tolerant-NILs were obtained from the Plant Breeding, Genetics and Biotechnology Division of the International Rice Research Institute (IRRI), Los Banos, Philippines. Seeds of the Korean rice cultivars were obtained from the Rice Research Division of the National Institute of Crop Science (NICS), Rural Development Administration (RDA), Republic of Korea. 'Nonglimna1', 'GL33', 'Moroberekan' and 'Ilpumbyeo' were also used to analyze genetic diversity using SSR markers.

\section{Management of drought stress and non-stress trials in tropical region}

In the lowland drought stress trials, seeds were sown in the nursery and 21-day-old seedlings were transplanted to the main field of IRRI, Philippines, during the 2011 and 2012 dry seasons. One seedling was transplanted per hill at a spacing of $10 \mathrm{~cm}$ between hills in a row. In non-stress trials, after transplanting, $\sim 5 \mathrm{~cm}$ of standing water was maintained in the field until drainage before harvest while in stress trials, stress was imposed by draining water from the paddy at 30 DAT (days after transplanting) and withholding irrigation until the soil moisture tension reached $-50 \mathrm{kPa}$ at $20 \mathrm{~cm}$ depth. Fields were then re-irrigated by flash flooding, and drained again after $\sim 24 \mathrm{~h}$. This cycle was repeated until harvest. Severe leaf rolling was observed during each stress period. All the experiments were laid out in an alpha lattice design and consisted of two replications. Spacing between rows was $0.25 \mathrm{~cm}$ in all trials. Plots were single-row in the selection experiments but mostly two-row in the evaluation experiments. Length of the plot was $5 \mathrm{~m}$ in lowland. Recommended levels of fertilizer were added, and weeds and pests were controlled when necessary. Days to flowering (DTF), plant height $(\mathrm{PH})$, grain yield of rough rice (Yield; $\mathrm{kg} / 10 \mathrm{a}$ ) were estimated as drought tolerance index. More experimental details can be found in Venuprasad et al. (2007 and 2008).

\section{Evaluation of agricultural traits in temperate region}

Seven drought tolerant-NILs including five Korean rice cultivars also were planted in a four-row plot with 35 plants per row by $30 \times 15 \mathrm{~cm}$ spacing in a randomized complete block design with three replications and evaluated for agronomic traits in the rice experimental plot of NICS, Cheolwon, Korea, using the standard evaluation method of rice (RDA 2003). For each line, five plants in the middle rows were used to determine heading date (HD), culm length (CL), panicle number per plant (PN), panicle length (PL), number of spikelet per panicle (NSP), percent of ripened grain (PRG), 1,000-grain weight of the brown rice $(\mathrm{GW})$, and grain yield of rough rice (Yield; kg/10a).

\section{SSR marker and genetic diversity analysis}

The 16 rice genotypes including drought tolerant-NILs were genotyped using 125 SSR markers distributed evenly on the 12 chromosomes to generate a dendrogram. Seventeen SSR markers flanking the DTY1.1, DTY2.2 and DTY3.1 regions were used for detecting polymorphic markers between recipients and donors. Genomic DNA was extracted from the fresh frozen leaves of the rice cultivars by a modified CTAB method (Rogers and Bendich, 1988). The procedure used in the PCR assay was as described by Suh et al. (2009). The PCR products were detected using 4\% denaturing polyacrylamide gel electrophoresis with silver staining. The DNA bands generated were analyzed and scored 1 (present) or 0 (absent) for each allele. The similarity matrices obtained using the simple matching coefficient were subjected to unweighed pair-group methods using arithmetic average (UPGMA) clustering and represented in dendrogram form using NTSYS-pc program (Rohlf 1993). Two Korean rice cultivars (Jinmibyeo and Hanareumbyeo) and only three QTL donors were used for parental polymorphism survey.

\section{RESULTS}

\section{Drought tolerance of Korean rice cultivars and drought tolerant-NILs under tropical conditions}

Seven drought tolerant-NILs including five Korean rice cultivars were screened under irrigated non-stress and drought stress at IRRI, Philippines during the 2011 and 
2012 dry seasons. Under drought stress, all drought tolerant-NILs showed higher grain yield than IR64 and the Korean rice cultivars (Table 3). In particular, IR86918-B439-B had the highest yield followed by IR77298-14-1-2 and IR81896-B-B-431 under drought stress. Among the five Korean cultivars, Hanareumbyeo yielded the highest followed by Sangnambatbyeo but their yields were lower than those of drought tolerant-NILs (Table 3). Three drought tolerant-NILs, IR86918-B-305-B (DTY 1.1), IR77298-14-1-2 (DTY2.2), IR81896-B-B-431 (DTY3.1) were selected for transferring the drought-QTLs to Korean rice cultivars, Jinmibyeo and Hanareumbyeo (Table 3).

Table 3. Morpho-agronomic traits and yield performance of Korean rice varieties and drought tolerant QTL-NILs under irrigated non-stress and mild-moderate drought stress conditions in 2011-2012 dry season at IRRI.

\begin{tabular}{|c|c|c|c|c|c|c|}
\hline \multirow{2}{*}{ Variety } & \multicolumn{3}{|c|}{ Irrigated non-stress ${ }^{z}$} & \multicolumn{3}{|c|}{ Drought stress } \\
\hline & DTF (days) & $\mathrm{PH}(\mathrm{cm})$ & Yield $(\mathrm{kg} / 10 \mathrm{a})$ & DTF (days) & $\mathrm{PH}(\mathrm{cm})$ & Yield $(\mathrm{kg} / 10 \mathrm{a})$ \\
\hline Jinmibyeo & 69 & 84 & $386 a b$ & 80 & 68 & $104 \mathrm{a}$ \\
\hline Unkwangbyeo & 63 & 76 & $354 a$ & 74 & 70 & $106 a$ \\
\hline Gayabyeo & 76 & 85 & $487 \mathrm{c}$ & 84 & 72 & $157 \mathrm{a}$ \\
\hline Hanareumbyeo & 77 & 81 & $616 \mathrm{e}$ & 85 & 66 & $242 \mathrm{c}$ \\
\hline Sangnambatbyeo & 74 & 92 & $366 a$ & 78 & 74 & $218 \mathrm{c}$ \\
\hline IR $86918-B-223-B$ & 75 & 149 & $544 d$ & 82 & 141 & $352 \mathrm{de}$ \\
\hline IR $86918-B-305-B$ & 75 & 156 & $543 d$ & 78 & 133 & $328 \mathrm{~d}$ \\
\hline IR $86918-B-382-B$ & 75 & 129 & $563 d$ & 81 & 117 & 356de \\
\hline IR $86918-B-439-B$ & 74 & 137 & $653 e$ & 78 & 122 & $380 \mathrm{e}$ \\
\hline IR 77298-14-1-2 & 83 & 84 & $533 d$ & 89 & 71 & $373 \mathrm{e}$ \\
\hline IR 81896-B-B-431 & 93 & 93 & $629 \mathrm{e}$ & 99 & 63 & $360 \mathrm{e}$ \\
\hline IR $84984-83-15-18-B-B$ & 84 & 125 & $573 \mathrm{de}$ & 87 & 105 & $309 d$ \\
\hline IR 64 & 75 & 104 & $569 d$ & 87 & 73 & $130 \mathrm{a}$ \\
\hline
\end{tabular}

${ }^{z}$ DTF: Days to flowering, PH: Plant height, Yield: Grain yield of rough rice. Means followed by the same letter are not significant at the $5 \%$ significance level by the least significant difference test (LSD $=0.05)$.

Table 4. Yield performance and agronomic traits of drought tolerant-NILs under irrigated non-stress condition in Korea.

\begin{tabular}{|c|c|c|c|c|c|c|c|c|}
\hline Variety & ${ }^{\mathrm{z}} \mathrm{HD}$ (M.D) & $\mathrm{CL}(\mathrm{cm})$ & PL $(\mathrm{cm})$ & $\mathrm{PN}$ & NSP & PRG (\%) & GW (g) & Yield $(\mathrm{kg} / 10 \mathrm{a})$ \\
\hline Jinmibyeo & 7.30 & 69 & 20 & 12 & 68 & 84.5 & 22.07 & $616 \mathrm{~d}$ \\
\hline Unkwangbyeo & 7.30 & 62 & 20 & 11 & 80 & 80.0 & 23.97 & $699 \mathrm{e}$ \\
\hline Hanareumbyeo & 8.10 & 68 & 21 & 10 & 75 & 91.9 & 24.83 & $707 \mathrm{e}$ \\
\hline Gayabyeo & 8.7 & 75 & 21 & 10 & 85 & 75.2 & 23.92 & $624 d$ \\
\hline Sangnambatbyeo & 8.7 & 75 & 24 & 9 & 81 & 80.2 & 21.01 & $518 \mathrm{bc}$ \\
\hline IR86918-B-223-B & 7.28 & 116 & 23 & 12 & 51 & 80.7 & 18.54 & $344 a$ \\
\hline IR86918-B-305-B & 8.10 & 128 & 24 & 12 & 66 & 81.4 & 20.20 & $487 \mathrm{~b}$ \\
\hline IR86918-B-382-B & 8.4 & 113 & 21 & 12 & 53 & 68.1 & 29.31 & $461 \mathrm{~b}$ \\
\hline IR86918-B-439-B & 8.9 & 106 & 27 & 10 & 72 & 74.8 & 26.28 & $561 \mathrm{c}$ \\
\hline IR77298-14-1-2 & 8.10 & 84 & 26 & 12 & 83 & 63.5 & 25.88 & $606 \mathrm{~d}$ \\
\hline IR81896-B-B-431 & \multicolumn{8}{|c|}{ Not flowering } \\
\hline IR84984-83-15-18-B-B & 8.4 & 103 & 21 & 11 & 90 & 86.4 & 21.31 & $512 \mathrm{bc}$ \\
\hline
\end{tabular}

${ }^{7}$ HD: Heading date(M: month, D: day), CL: Culm length, PL: Panicle length, PN: Panicle number per plant, NSP: Number of spikelet per panicle, PRG: Percent of ripened grain, GW: 1,000 grain weight of brown rice, Yield: Grain yield of rough rice. Means followed by the same letter are not significant at the 5\% significance level by the least significant difference test $(\mathrm{LSD}=0.05)$. 
Jinmibyeo and Hanareumbyeo were selected as recipient parents because these rice cultivars are well adapted in temperate and tropical regions.

\section{Yield potential of drought tolerant-NILs under non- stress temperate conditions}

Seven drought tolerant-NILs including five Korean rice cultivars were also evaluated for yield potential under irrigated non-stress experimental plot at Cheolwon substation, NICS, Korea in 2012. The agronomic traits and yield performance of IR81896-B-B-431 could not be evaluated because it does not flower in Korean environmental conditions. All Korean rice cultivars except Sangnambatbyeo showed higher yield than those of drought tolerant-NILs collected from IRRI (Table 4). It is likely that the lower yield potential of the drought tolerant-NILs comparison with the Korean rice cultivars is related to their selection under tropical environmental conditions, and hence their lack of adaptation to temperate conditions. However, IR77298-14-1-2 showed similar yield potential to the Korean rice cultivars. Thus, IR77298-14-1-2 may be the best adapted donor for introducing drought tolerance QTL into temperate japonica rice.

\section{Parental polymorphism survey for foreground and background selection markers}

The three drought tolerant-NILs, IR86918-B-305-B (DTY1.1), IR77298-14-1-2 (DTY2.2), IR81896-B-B-431

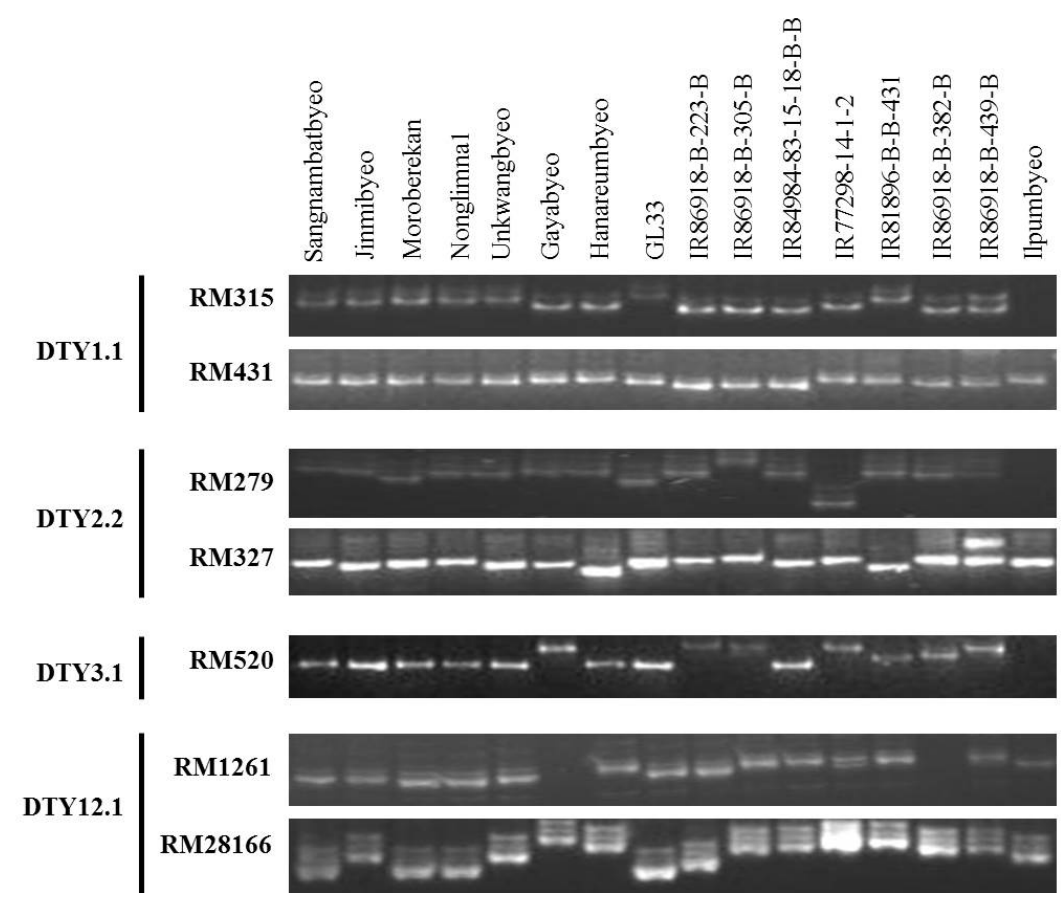

Fig. 1. PCR assay for amplification of identified QTL regions using SSR markers linked with QTLS for drought tolerance on chromosomes $1,2,3$, and 12 .

Table 5. Flanking SSR markers for drought-QTL region and DNA polymorphism between donor and recurrent parents.

\begin{tabular}{lcccc}
\hline \hline \multirow{2}{*}{ Donor parent } & QTLs & \multicolumn{2}{c}{ Polymorphism (\%) } & Flanking markers \\
\cline { 3 - 4 } & & Jinmibyeo & Hanareumbyeo & RM431, RM11943, RM12023, \\
IR86918-B-305-B & $\left(D T Y_{1.1}\right)$ & 70 & 46 & RM12233, RM12146, RM12091 \\
IR77298-14-1-2 & $\left(D T Y_{2.2}\right)$ & 63 & 37 & RM109, RM279, RM236 \\
IR81896-B-B-431 & $\left(D T Y_{3.1}\right)$ & 66 & 28 & RM520, RM416, RM16030 \\
\hline
\end{tabular}


(DTY3.1), were used as the donor parents for drought tolerance, and Jinmibyeo and Hanareumbyeo were used as the recipient parents. The parental polymorphism survey was performed with 17 SSR markers flanking the target QTLs on two of the Korean rice cultivars and three of the drought tolerant-NILs. Twelve polymorphic markers were identified between recipient and donor parents at target QTL regions for drought tolerance (Table 5 and Fig. 1). The SSR markers, RM431, RM11943, RM12023, RM12233, RM12146 and RM12091 on chromosome 1 showed polymorphism between two Korean rice cultivars and IR86918-B-305-B in DTY1.1 region. The SSR markers, RM109, RM279 and RM236 on chromosome 2 showed polymorphism between two of the Korean rice cultivars and IR77298-14-1-2 in DTY2.2 region. The SSR markers, RM520, RM416 and RM16030 on chromosome 3 showed polymorphism between two of the Korean rice cultivars and IR81896-B-B-431 in DTY3.1 region. SSR markers that showed polymorphism between the two recipient parents and three donor parents around the target drought-QTLs were used as flanking markers to introduce the drought-QTLs of donors (Table 5).

\section{Genetic divensity of drought tolerant lines using SSR markers}

Cluster analysis for the 16 rice germplasm was performed based on similarity coefficient matrices calculated from 125 SSR markers to generate a dendrogram (Fig. 2). The sixteen rice cultivars could be separated into two main clusters such as japonica and indica groups. Four rice cultivars (Sangnambatbyeo, Nonglimna1, GL33, Moroberekan) were sub-grouped in japonica cluster. Sangnambatbyeo, Nonglimna1 and Moroberekan are upland japonica rice and GL33 is a japonica weedy rice.

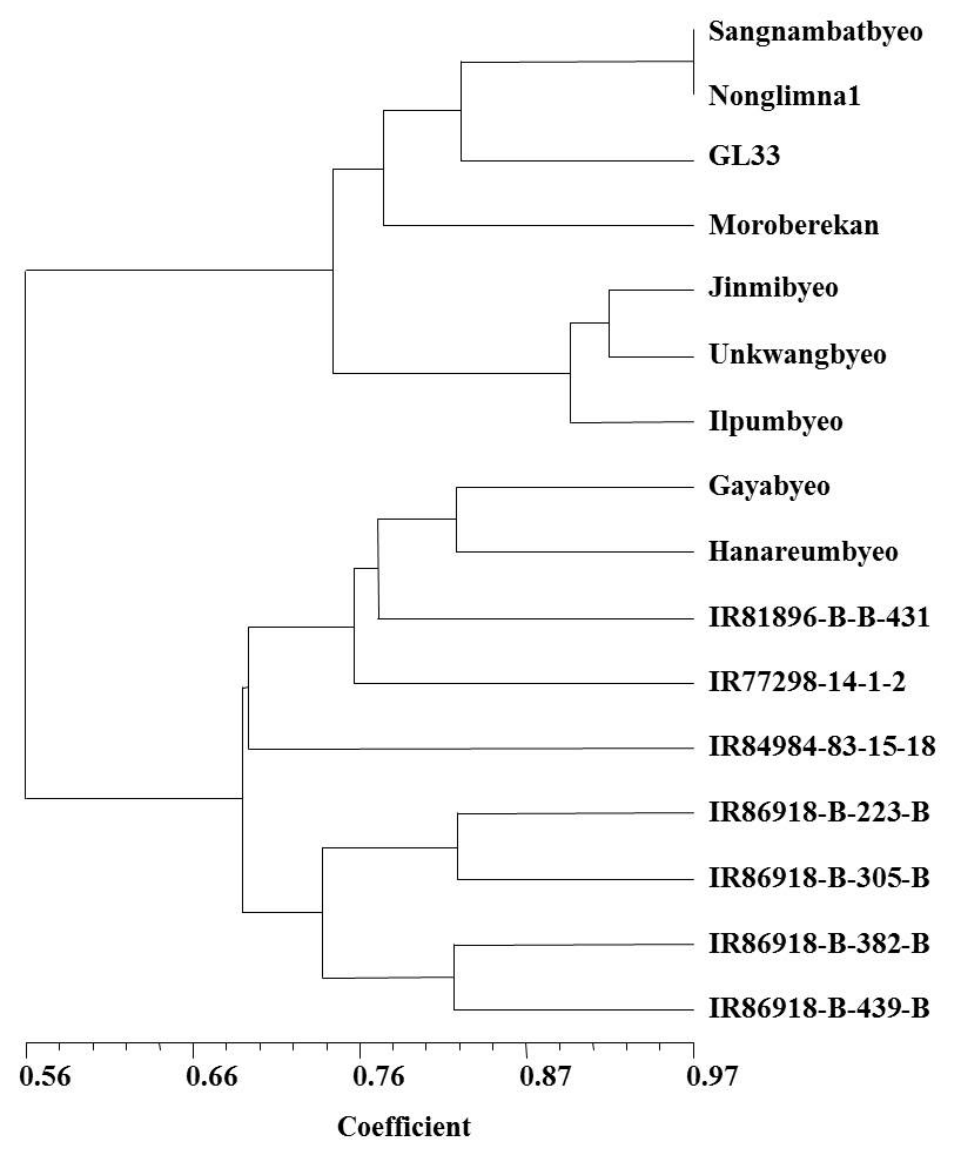

Fig. 2. Dendrogram of nine Korean rice germplasm and seven donors for drought tolerance clustered by the similarity coefficients of 125 SSR markers. 


\section{DISCUSSION}

As rainfed environments are highly variable, breeding rice for drought-prone rainfed ecosystems requires genotypes that combine high yielding ability with good drought tolerance in addition to tolerance to prevalent biotic stresses. The molecular breeding program for drought at IRRI has identified several QTLs for grain yield under drought (Bernier et al. 2007; Venuprasad et al. 2009a; Vikram et al. 2011; Dixit et al. 2012). The present study evaluated the grain yield of drought tolerant-NILs under irrigated non-stress and drought stress conditions and identified polymorphic SSR markers between Korean rice cultivars and drought tolerant-NILs to introduce the drought QTLs into Korean rice cultivars.

All drought tolerant-NILs showed higher grain yield than those of IR64 and Korean rice cultivars under drought stress in tropical conditions. IR86918-B-439-B yielded highest followed by IR77298-14-1-2 and IR81896-B-B-431 under drought stress. IR86918-B-439-B possessed drought tolerance QTL, DTY1.1 from N22 in IR64 genetic background (Vikram et al. 2011). The drought tolerance QTL, DTY1.1 had a major positive effect for grain yield under reproductive-stage drought stress and non-stress conditions. The effect of this QTL is also consistent in the different genetic backgrounds, Swarna and MTU1010 (Vikram et al. 2011). IR77298-14-1-2 is nearly isogenic to IR64 but differs in its tolerance to drought and tungro disease. It has a drought tolerance QTL, DTY2.2 from Adaysel in IR64 genetic background (Swamy et al. 2011; Swamy et al. 2013). The drought tolerance QTL, DTY2.2 has high and consistent additive effect under severe lowland drought stress (Dixit et al. 2012). QTLs for root length and thickness adjacent to the qDTY2.2 have been reported (Champoux et al. 1995; Kamoshita et al. 2002; MacMillan et al. 2006). IR77298-14-1-2 is a valuable breeding line because it not only has drought tolerance but also tungro resistance (Venuprasad et al. 2007). IR81896B-B-431 has the drought tolerance QTL, DTY3.1 from Apo in a Swarna genetic background (Venuprasad et al. 2009a). DTY3.1 had a large effect on grain yield under severe lowland drought stress. It also explained considerable variance for yield under mild stress in lowland conditions and aerobic environments (Venuprasad et al. 2009a).

Six SSR markers showed polymorphism between two Korean rice cultivars and IR86918-B-305-B in the region of DTY1.1. This region has been identified between RM431 and RM11943 on chromosome 1 in the background of highly popular elite rice varieties showing consistent effects against different genetic backgrounds (Vikram et al. 2011). RM431 and RM11943 markers also showed polymorphism between two Korean rice cultivars and IR86918-13-305-B in this study. These markers are very useful as flanking markers for DTY1.1 introduction. Three SSR markers, RM109, RM279, and RM236 showed polymorphism between two of the Korean rice cultivars and IR77298-14-1-2 in the region of DTY2.2. DTY2.2 was identified between RM279 and RM555 having an effect on grain yield under severe stress conditions by CIM analysis (Dixit et al. 2012). The SSR marker RM279 was found polymorphic for the two Korean rice cultivars and IR7729814-1-2. Three SSR markers were also polymorphic between two of the Korean rice cultivars and IR81896-B-B-431 in the region of DTY3.1. The peak of DTY3.1 was located at $10.0 \mathrm{cM}$ and flanked by RM520 $(9.1 \mathrm{cM})$ and RM416 (10.0cM) (Venuprasad et al. 2009a). Moreover, RM520 and RM416 were also found polymorphic between the two Korean rice cultivars and IR81896-B-B-431. These markers will therefore be useful as flanking markers to introduce the DTY3.1 into Korean rice cultivars. The group of polymorphic SSR markers at the target drought QTLs, DTY1.1, DTY2.2 and DTY3.1 can be utilized to introduce those QTLs into Korean rice cultivars as foreground selection markers.

The sixteen rice cultivars could be separated into two main clusters corresponding the japonica and indica groups following cluster analysis using SSR marker results. The lines IR77298, IR81896 and IR84984 sub-grouped with the Korean Tong-il cultivars. The cultivars Sangnambatbyeo, Nonglimna1, GL33 and Moroberekan sub-grouped in the japonica cluster. The cultivars Sangnambatbyeo, Nonglimna1, GL33, and Moroberekan were slightly genetically different from the japonica rice cultivars because those lines are upland or japonica weedy rice. The four IR86918 lines sub-grouped within the indica group. These lines are drought tolerant-NILs derived from a cross IR64 (recipient) and N22 (donor for drought tolerance) by backcrossing 
(Kumar 2011; Vikram et al. 2011), and as expected from their pedigree, they are genetically very similar. Although lines developed from IR77298 were derived from a cross between IR64 and Adaysel, they were genetically distinct from the IR86918 lines but more closely related to the Tong-il cultivars from Korea. This suggests that the lines derived from IR77298 did not recover as much of the IR64 genetic background as the IR86918 lines. Lines derived from IR81896 and IR84984 also sub-grouped with Korean Tong-il cultivars. These lines are drought tolerant-NILs from crosses between Swarna, Vandana (recipients) and Apo, Way Rarem (donors for drought tolerance). IR77298 and IR84984 lines also demonstrated desirable agronomic traits and yield potential under temperate environmental conditions in Korea. This suggests that it may be more efficient to introduce the drought tolerance QTLs of those lines into the background of Korean Tong-il types in temperate regions. Varieties with an improved drought tolerance and high yield potential are likely to reduce production risk and contribute to food security through more stable production when affected by drought.

\section{ACKNOWLEDGMENT}

This work was carried out with the support of RDA-IRRI international special collaborative project 'Development drought-tolerance japonica rice using marker-assisted backcrossing' and Cooperative Research Program for Agriculture Science \& Technology Development (Project No. PJ009325062014) Rural Development Administration, Republic of Korea and research project of Korea Institute of Planning \& Evaluation for Technology in Food, Agriculture, Forestry \& Fisheries (iPET) (Code no. 408529-03-3-SB020), Republic of Korea.

\section{REFERENCES}

Bates BC, Kundzewicz ZW, Wu S, Palutikof JP. 2008. Climate change and water. In: Technical paper of the Intergovernmental Panel on Climate Change, IPCC Secretariat, Geneva.
Bernier J, Kumar A, Venuprasad R, Spaner D, Atlin GN. 2007. A large-effect QTL for grain yield under reproductive stage drought stress in upland rice. Crop Sci. 47: 505- 516.

Champoux C, Wang G, Sarkarung S, Mackill DJ, O'Toole JC, Huang N, McCouch SR. 1995. Locating genes associated with root morphology and drought avoidance in rice via linkage to molecular markers. Theor. Appl. Genet. 90: 969-981.

Dixit S, MallikejunA Swamy BP, Vikram P, Ahmed HU, Sta Cruz MT, Amante M, Atri D, Leung H, Kumar A. 2012. Fine mapping of QTLs for rice grain yield under drought reveals sub-QTLs conferring a response to variable drought severities. Theor. Appl. Genet. 125: 155-169.

Kamoshita A, Wade LJ, Ali ML, Pathan MS, Zhang J, Sarkarung S, Nguyen HT. 2002. Mapping QTLs for root morphology of a rice population adapted to rainfed lowland conditions. Theor. Appl. Genet. 104: 880-893.

Kumar A, Bernier J, Verulkar S, Lafitte HR, Atlin GN. 2008. Breeding for drought tolerance: direct selection for yield, response to selection and use of drought- tolerant donors in upland and lowland-adapted populations. Field Crops Res. 107: 221-231.

Kumar A. 2011. Making rice less thirsty: progress at IRRI. Annual Seminar series, March 3, 2011.

Maclean JL, Dawe DC, Hardy B, Hettel GP. 2002. Rice Almanac, third ed. IRRI, Los Banos, Philippines, p. 253.

MacMillan K, Emrich K, Piepho HP, Mullins CE, Price AH. 2006. Assessing the importance of genotype $\times$ environmental interaction for root traits in rice using a mapping population. II. Conventional QTL analysis. Theor. Appl. Genet. 113: 953-964.

Pandey S, Bhandari HN, Hardy B. 2007. Economic costs of drought and rice farmers' coping mechanisms. A Cross-Country Comparative Analysis. International Rice Research Insti-tute/World Scientific Publishing, Los Banos, Philippines/Singapore, pp. 1-9.

RDA (Rural Development Administration). 2003. Manual for standard evaluation method in agricultural experiment and research. RDA, Suwon (Korea). p. 838.

Rogers OS, Bendich AJ. 1988. Extraction of DNA from plant tissues. Plant Mol. Biol. Manual A6 1-10.

Rohlf FJ. 1993. NTSYS-PC numerical taxonomy and multivariate analysis system. Version 2.0j. Exter. Publ. Setauket, New York. 
Suh JP, Roh JH, Cho YC, Han SS, Kim YG, Jena KK. 2009.

The Pi40 gene for durable resistance to rice blast and molecular analysis of Pi40-advanced backcross breeding lines. Phytopathology 99: 243-250.

Swamy BPM, Ahmed HU, Henry A, Mauleon R, Dixit S, Vikram P, Tilatto R, Verulkar SB, Perraju P, Mandal NP, Variar M, Robin S, Chandrababu R, Singh ON, Dwivedi JL, Das SP, Mishra K, Yadaw RB, Aditya TL, Karmakar B, Satoh K, Moumeni A, Kikuchi S, Leung H, Kumar A. 2013. Genetic, physiological and gene expression analyses reveal that multiple QTL enhance yield of rice mega-variety IR64 under drought. PLOS ONE 8p. e62795.

Swamy BPM, Vikram P, Dixit S, Ahmed HU, Kumar A. 2011. Meta-analysis of grain yield QTL identified during agricultural drought in grasses showed consensus. BMC Genomics 12: 319.

Venuprasad R, Bool ME, Dalid CO, Bernier J, Kumar A, Atlin GN. 2009b. Genetic loci responding to two cycles of divergent selection for grain yield under drought stress in a rice breeding population. Euphytica 167: 261-269.

Venuprasad R, Dalid CO, Del Valle M, Zhao D, Espiritu M, Sta Cruz MT, Amante M, Kumar A, Atlin GN. 2009a.
Identification and characterization of large-effect quantitative trait loci for grain yield under lowland drought stress in rice using bulk-segregant analysis. Theor. Appl. Genet. 120: 177-190.

Venuprasad R, Sta Cruz MT, Amante M, Magbanua R, Kumar A, Atlin GN. 2008. Response to two cycles of divergent selection for grain yield under drought stress in four rice breeding populations. Field Crops Res. 107: 232-244.

Venuprasad R, Zenna N, Choi IR, Amante M, Virk PS, Kumar A. 2007. Identification of marker loci associated with tungro and drought tolerance in near-isogenic rice lines derived from IR64/Aday Sel. IRRN. 32.1: 27-29.

Vikram P, MallikejunA Swamy BP, Dixit S, Ahmed HU, Sta Cruz MT, Sing AK, Kumar A. 2011. qDTY1.1, a major QTL for rice grain yield under reproductive-stage drought stress with a consistent effect in multiple elite genetic backgrounds. BMC Genetics 12: 89.

Wassmann R, Jagadish SVK, Sumfleth K, Pathak H, Howell G, Ismail A, Serraj R, Redona E, Singh RK, Heuer S. 2009. Regional vulnerability of climate change impacts on Asian rice-production and scope for adaptation. Adv. Agron. 102: 91-133. 